\title{
Reversible Pore Gating in Aqueous Mixtures via External Potential
}

\author{
Sela Samin \\ Institute for Theoretical Physics, Center for Extreme Matter and Emergent Phenomena, \\ Utrecht University, Princetonplein 5, 3584 CC Utrecht, The Netherlands \\ Yoav Tsori \\ Department of Chemical Engineering and the Ilse Katz Institute for Nanoscale Science and Technology, \\ Ben-Gurion University of the Negev, 84105 Beer-Sheva, Israel.
}

(Dated: April 20, 2016)

\begin{abstract}
We examine theoretically porous membranes in aqueous mixtures. We show that large membrane pores can be reversibly gated from 'off' (co-solvent-rich, poor conductor of ions and other solutes) to 'on' (water-rich, good conductor) states by an external potential. The transition voltage or charge for switching depends on the membrane hydrophilicity/hydrophobicity, the salt content, the preferential solvation of the salt ions, and the temperature. These parameters also determine whether the filling transition is abrupt or gradual.
\end{abstract}

Transport of liquids and solutes across nano- and mesoscale pores occurs in many biological and synthetic membranes [1, 2]. Considerable research on the pore chemistry and shape has been carried out to improve the membrane's conductivity and selectivity and to prevent the membrane from clogging [3]. In pore gating, electric fields have advantages over the use of thermal variations [4, 5] or pressure difference across the membrane [6] since they can be easily switched on and off and do not require the membrane to be mechanically robust. Current works consider electric fields that are parallel to the pore's axis and pure water or coexistence of vapor and water [ $7-10]$.

Here we propose a novel methodology to reversibly gate hydrophobic or hydrophilic pores by using aqueous solutions and fields that are perpendicular to the pore's walls. For hydrophobic membranes, the pore opens and fills with water when voltage is applied to the membrane. The pore closes by filling with the co-solvent by natural diffusion on a time that scales as the pore size squared once the voltage is removed. Ionic current ceases a short time after turning on or off the voltage.

The pore filling described below is promoted by the preferential solvation of ions in water, which has previously been shown to modify bulk coexistence [11 13 , solvent and ion adsorption on surfaces [11 13], and the inter-particle potential in colloidal suspensions [14 20]. Here, a purely solvation induced transition in confinement is quantified for the first time, and it is shown that its magnitude is comparable to that of capillary condensation. More importantly, the filling transition is predicted to occur even for highly hydrophobic pores, unlike capillary condensation. Even more so, continuous filling by an external potential is predicted for hydrophobic pores far above the mixture critical temperature.

We consider a small pore in a membrane embedded in a large reservoir of aqueous mixture. For large membrane potentials the ion density at the surfaces becomes very high and therefore we use a Modified Poisson-Boltzmann (MPB) approach [21] employing the incompressibility constraint $\phi+\phi_{\mathrm{cs}}+v_{0} n^{-}+v_{0} n^{+}=1$, where $\phi$ and $\phi_{\mathrm{cs}}$ are the volume fractions of water and cosolvent respectively, $v_{0}$ is the common molecular volume of all species, and $n^{ \pm}$ is the number density of cations and anions, respectively.

The solvent free energy density of mixing is

$$
f_{\mathrm{m}}=\frac{k_{B} T}{v_{0}}\left[\phi \log (\phi)+\phi_{\mathrm{cs}} \log \left(\phi_{\mathrm{cs}}\right)+\chi \phi \phi_{\mathrm{cs}}\right] .
$$

$k_{B} T$ is the thermal energy and $\chi \sim 1 / T$ is the Flory interaction parameter. Eq. (1) leads to an Upper Critical Solution Temperature type phase diagram. In the $\phi-T$ plane, a homogeneous phase is stable above the binodal curve, $\phi_{b}(T)$, whereas below it the mixture separates to water-rich and water-poor phases with compositions given by $\phi_{b}(T)$. The two phases become indistinguishable at the critical point $\left(\phi_{c}, \chi_{c}\right)=(1 / 2,2)$.

The free energy density of the ions dissolved in the mixture, $f_{\mathrm{i}}$, is modeled as

$$
\begin{aligned}
f_{\mathrm{i}}= & k_{B} T\left[n^{+} \log \left(v_{0} n^{+}\right)+n^{-} \log \left(v_{0} n^{-}\right)\right. \\
& \left.-\phi\left(\Delta u^{+} n^{+}+\Delta u^{-} n^{-}\right)\right]
\end{aligned}
$$

The first line in Eq. (2) is the entropy of the ions and the second line models the solvent specific short-range interactions between ions and solvents, where the solvation parameters, $\Delta u^{ \pm}$, measure the preference of ions towards a local water environment [11, 18, 22]. We choose a simple linear solvation model for clarity. Nonetheless, we note that our results remain qualitatively the same and quantitatively similar when more complex solvation models are employed, such as the one offered by Bier et al. [13].

Experiments show that $\Delta G_{t}$, the Gibbs transfer energy for transferring an ion from a solvent with composition $\phi_{1}$ to a solvent with composition $\phi_{2}$ is on the order of $1-10 k_{B} T$ in aqueous mixtures [23] and since $\Delta G_{t}=$ $k_{B} T \Delta u^{ \pm}\left(\phi_{2}-\phi_{1}\right)$ one finds that $\Delta u^{ \pm} \sim 1-10$. In general $\Delta G_{t}$ is highly ion specific and also depends strongly on the ion sign and valency. The filling transition is robust; 
for clarity of presentation we present only the simple case where $\Delta u^{+}=\Delta u^{-}$.

The electrostatic energy density, $f_{\mathrm{e}}$, for a monovalent salt is given by

$$
f_{\mathrm{e}}=-\frac{1}{2} \varepsilon(\phi)(\nabla \psi)^{2}+e\left(n^{+}-n^{-}\right) \psi
$$

where $\psi$ is the electrostatic potential, $e$ is the elementary charge and $\varepsilon(\phi)$ is the permittivity, assumed to depend linearly on the mixture composition: $\varepsilon(\phi)=$ $\varepsilon_{\mathrm{cs}}+\left(\varepsilon_{w}-\varepsilon_{\mathrm{cs}}\right) \phi$, where $\varepsilon_{w}$ and $\varepsilon_{\mathrm{cs}}$ are the water and cosolvent permittivities, respectively. This $\phi$ dependence of $\varepsilon$ leads in nonuniform electric fields to a dielectrophoretic force which attracts the high permittivity water towards charged surfaces [19, 24].

The surface energy density $f_{s}$ due to the contact of the mixture with a solid surface is given by:

$$
f_{s}=k_{B} T \Delta \gamma \phi\left(\mathbf{r}_{s}\right)+e \sigma \psi\left(\mathbf{r}_{s}\right)
$$

where $\mathbf{r}_{s}$ is a vector on the surface. The first term in Eq. (4) models the short-range interaction between the fluid and the solid. The parameter $\Delta \gamma$ measures the difference between the solid-water and solid-cosolvent surface tensions. The second term in Eq. (4) is the electrostatic energy when the surface carries a charge density $e \sigma$.

The pore is modeled as two identical flat plates with area $A$ located at $z= \pm D / 2$ (pore width is $D$ ). The pore walls can either carry a fixed surface charge density $e \sigma$ or have a constant potential $\psi_{s}$. The pore is in contact with a reservoir for which the electrostatic potential is $\psi=0$. The pore walls can be hydrophilic $(\Delta \gamma>0)$ of hydrophobic $(\Delta \gamma<0)$. In this planar geometry, the grand potential is given by

$$
\begin{aligned}
& \Omega\left[\phi(z), n^{ \pm}(z), \psi(z)\right]=A \int\left[\frac{1}{2} C(\nabla \phi)^{2}+f_{\mathrm{m}}+f_{\mathrm{i}}+f_{\mathrm{e}}\right. \\
& \left.-\lambda^{+} n^{+}-\lambda^{-} n^{-}-\mu \phi / v_{0}\right] \mathrm{d} z+2 A f_{s}
\end{aligned}
$$

The square-gradient term accounts for the energetic cost of composition inhomogeneities, where $C=k_{B} T \chi / v_{0}^{1 / 3}$ is a positive constant [17]. The chemical potentials of the cations and anions are $\lambda^{ \pm}$, respectively, and that of the mixture is $\mu$.

The pore's behavior follows from the profiles $\phi(z)$, $\psi(x)$, and $n^{ \pm}(x)$. The variational equations $\partial \Omega / \partial n^{ \pm}=0$ allow isolation of $n^{ \pm}$in the form of a Boltzmann-like distribution as a function of $\phi$ and $\psi$ (see Supplementary material). We solve numerically the two other equations $\partial \Omega / \partial \phi=0$ and Gauss's law $\nabla \cdot(\varepsilon(\phi) \nabla \psi)=e\left(n^{-}-n^{+}\right)$. The boundary conditions for $\phi$ at the walls are $\mathrm{d} \phi / \mathrm{d} z=$ $\Delta \gamma / C$ 25]. The condition for the potential is either $\mathrm{d} \psi / \mathrm{d} z=-e \sigma / \varepsilon(\phi)$ or $\psi=\psi_{s}$, corresponding to fixed charge density or potential, respectively.
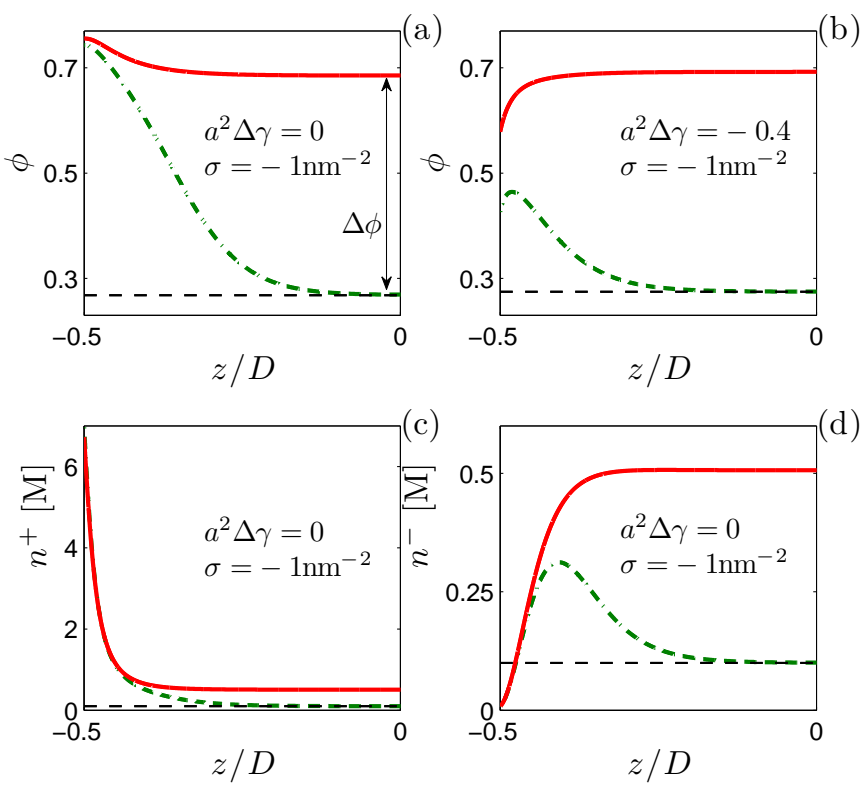

FIG. 1. Composition and ion density profiles for bulk compositions $\phi_{0}$ just before (dash-dot lines) and after (solid lines) the filling composition $\phi^{*}$. (a) Composition profiles for a charged and chemically indifferent pore (see figure for parameter values). The composition "jump" at the midplane of the pore is denoted by $\Delta \phi$. (b) A charged hydrophobic pore. (cd) the cation and anion density profiles corresponding to (a). The dashed lines in (a)-(b) correspond to $\phi_{0}$ and in (c)-(d) to $n_{0}$. We used $t=0.96, n_{0}=0.1 \mathrm{M}$. Unless otherwise stated, in all figures we took $D=10 \mathrm{~nm}, \Delta u^{ \pm}=4, \varepsilon_{w}=79.5, \varepsilon_{\mathrm{cs}}=6.9$ and $T_{c}=307$ K. $a=v_{0}^{1 / 3}=3.4 \AA$ is a molecular length.

We focus on homogeneous water-poor mixtures with a small enough average bulk composition $\phi_{0}$ : $\phi_{0}<\phi_{t}<\phi_{b}$. $\phi_{t}$ is the composition for which a bulk mixture first undergoes a so-called precipitation transition [12], where small water-rich droplet begin to nucleate due to the preferential solvation of ions in them. The derivation of $\phi_{t}$ is detailed in the Supplementary material. We find that in the vicinity of $\phi_{t}$ two types of configurations are possible in equilibrium, distinguished by the value of the composition at the pore midplane, $\phi(z=0)$ : either the pore has the bulk composition $\phi(z=0) \approx \phi_{0}<\phi_{c}$ or it fills with water and then $\phi(z=0)>\phi_{c}$. The stable configuration is the one for which the grand potential is lower. A first-order filling transition from one configuration to the other occurs when the corresponding grand potentials are equal at a composition $\phi_{0}=\phi^{*}$.

The composition and ion density profiles in the vicinity of the filling transition are shown in Fig. 1 for several scenarios. In this figure only the region $-D / 2 \leq z \leq 0$ of the symmetric profiles is shown. The reduced temperature is set to $t \equiv T / T_{c}=0.96$, where $T_{c}$ is the mixture critical temperature, and the salt is assumed hydrophilic, $\Delta u^{ \pm}=4$. Dash-dot and solid curves correspond to profiles just before and after the transition, 
respectively, while the horizontal dashed lines show the bulk values. A purely solvation-induced filling transition is demonstrated in Fig. 1 (a), where the pore walls are chemically indifferent, $\Delta \gamma=0$, but are highly charged. Here, prior to the transition, a wide adsorption layer is created when the counter-ions "drag" with them the water to the walls such that $\phi(z=-D / 2)>\phi_{c}=1 / 2$. The thickness of the layer here is associated with a modified Debye length $\lambda_{D}\left(n_{0} ; \phi_{0}, \Delta u\right)$ (For more details see Ref. [17]). At the transition, the composition profile jumps discontinuously to $\phi(z)>\phi_{c}$ throughout the pore volume.

The corresponding ion profiles in Fig. 1 (c)-(d) show that the ion density decays to its bulk value closer to the wall than the composition because composition gradients are energetically costly. Therefore, farther from the wall the relevant length scale is a modified bulk correlation length, $\xi\left(T ; n_{0}, \Delta u\right)$, associated with the width of interfaces and depending strongly on $T-T_{c}$ [17]. The co-ions are electrostatically repelled from the wall but are also drawn to it due to their favorable solvation in water, resulting in a maximum in the co-ions profile, shown in Fig. 1 (d). Close enough to the binodal it is energetically favorable to eliminate the large composition gradients and the filling transition ensues. We stress that once filling takes place the profiles do not decay to the bulk values far from the wall.

The filling transition is predicted to occur even for a charged but highly hydrophobic pore with $a^{2} \Delta \gamma=-0.4$. Fig. 1 (b) shows the composition profiles for this case, where although the solvent is depleted close to the wall (evidenced by the positive slope $\phi^{\prime}(z=-D / 2)$ ), the attraction of the counter-ions to the walls together with the preferential solvation leads to an increase in the water composition and eventually to the filling of the pore. Although the short-range interaction of the solvent with the pore walls can also promote filling as in regular capillary condensation, here the importance of selective solvation, which is a volume contribution to the free energy, is much larger. This holds when the pore walls are highly charged, the salt concentration is large and the coupling with the solvent is strong.

The stability of the mixture in the pore is determined by the filling curve $\phi^{*}(T)$ in the $\phi_{0}-T$ plane. This curve is the value of the bulk composition $\phi_{0}$ for which filling occurs, for different temperatures. The filling curve for $D=10 \mathrm{~nm}, n_{0}=0.1 \mathrm{M}$ and $\Delta u^{ \pm}=4$ is shown in Fig. 2. We plot $\phi^{*}(T)$ for a highly charged and chemically indifferent pore (solid, $a^{2} \Delta \gamma=0, \sigma=-1 \mathrm{~nm}^{2}$ ) and an uncharged and hydrophilic pore (dashed, $a^{2} \Delta \gamma=0.1$, $\sigma=0)$. For comparison, we also plot in Fig. 2 the capillary condensation curve of a salt-free mixture with hydrophilic walls (dash-dot, $a^{2} \Delta \gamma=0.1$ ). In all cases, the discontinuity of the physical quantities across the curve vanishes at a film critical point and increase with temperature decreasing away from it.

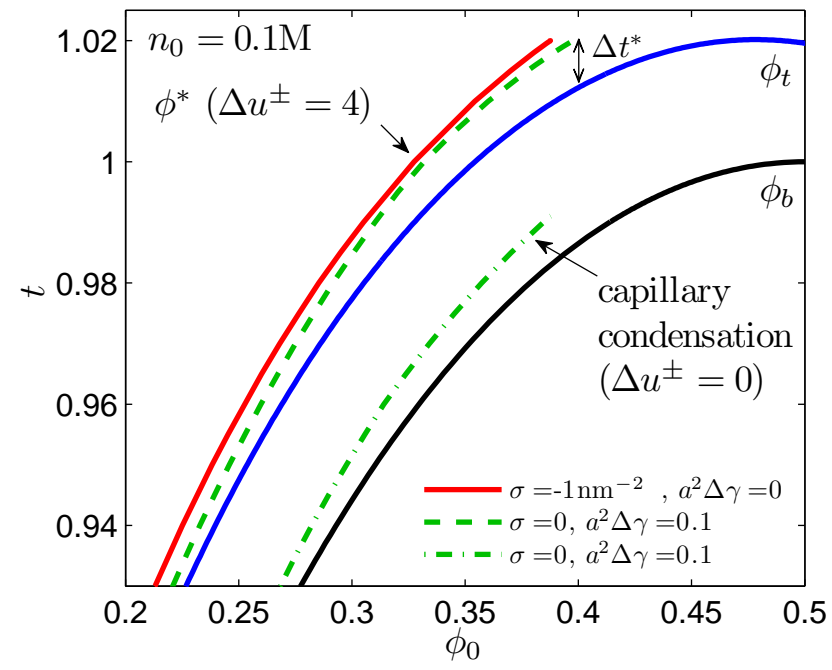

FIG. 2. The filling composition curve $\phi^{*}$ vs reduced temperature $t=T / T_{c}$ for membranes with pores of width $D=10$ $\mathrm{nm}$ embedded in aqueous mixtures with hydrophilic ions with $\Delta u^{ \pm}=4$. The finite volume of the ions is neglected. Topmost solid line (red): $\phi^{*}$ for a charged and chemically indifferent pore $\left(\sigma=-1 \mathrm{~nm}^{-2}, \Delta \gamma=0 a^{-2}\right)$. The filling effect is weaker when the pore is uncharged but hydrophilic $(\sigma=0$, $\left.\Delta \gamma=0.1 a^{-2}\right)$, dashed line. Solid curve denoted as $\phi_{t}$ is the mixing-demixing phase boundary in the bulk. The regular capillary condensation line for hydrophilic pores $\left(\Delta u^{ \pm}=0\right.$, $\left.\Delta \gamma=0.1 a^{-2}\right)$ is plotted as a reference (dash-dot line). $\phi_{b}$ is the bulk mixing-demixing binodal line in the absence of salt.

The temperature shift of the reciprocal filling curve $T^{*}\left(\phi_{0}\right)$ under confinement, $\Delta t^{*}$, is defined as

$$
\Delta t^{*}=t^{*}\left(\phi_{0} ; D\right)-t^{*}\left(\phi_{0} ; D \rightarrow \infty\right)=t^{*}\left(\phi_{0} ; D\right)-t\left(\phi_{t}\right)
$$

where $\phi_{t}(t)$ is the bulk mixing-demixing curve. $\Delta t^{*}$ quantifies the magnitude of the filling phenomena and should be readily accessible experimentally.

Based on the above insight we now turn to check whether indeed pore filling can be gated by a change of the external potential. Tuning of the potential can be realized by the use of activated carbon based membranes, as in e.g. supercapacitors. We find that the average water fraction $\langle\phi\rangle$ in a hydrophobic pore increases with increasing (negative) wall potential $\left|\psi_{s}\right|$ even when there is no discontinuous filling transition, see Fig. 3. At zero potential, $\langle\phi\rangle$ is negative as water is depleted from the pore. Clearly for $\left|\psi_{s}\right|>0.22 \mathrm{~V}\left(n_{0}=0.01 \mathrm{M}\right)$ or for $\left|\psi_{s}\right|>0.15 \mathrm{~V}\left(n_{0}=0.1 \mathrm{M}\right)$, water is a majority in the pore.

In Fig. 4 we plot $\Delta t^{*}$ vs the surface potential $\psi_{s}$ for a highly hydrophobic pore and for several values of $\Delta u$. Since the pore is hydrophobic the filling occurs only above a critical value for the potential and the magnitude of $\Delta t^{*}$ strongly depends on $\Delta u$. The value of the critical voltage (the value of $\left|\psi_{s}\right|$ of the bottom-left point of 


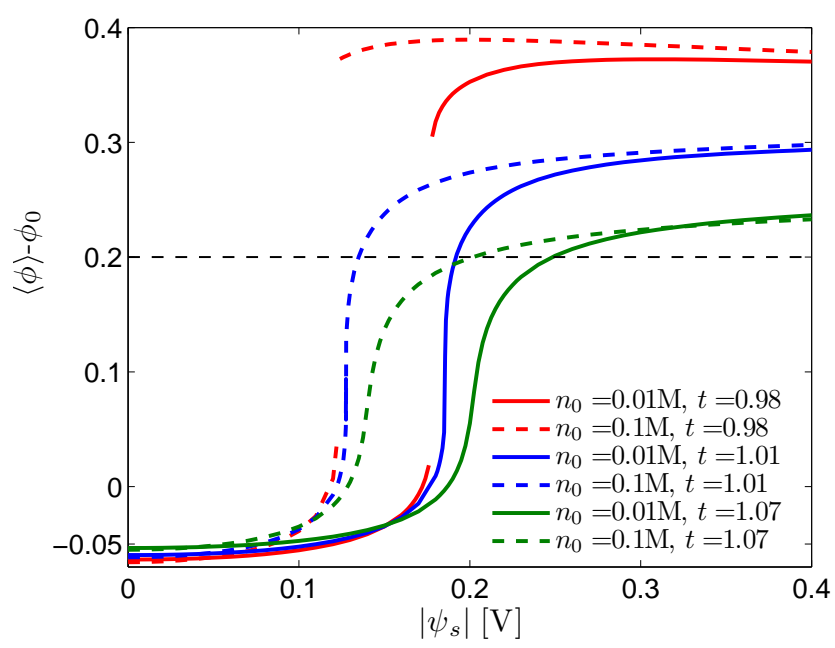

FIG. 3. Pore filling with external potentials. Curves show the difference between the average pore water fraction $\langle\phi\rangle$ and the bulk value $\phi_{0}$ vs pore potential $\left|\psi_{s}\right|$ for different salt content and temperatures. The filling is discontinuous for $t=0.98$ and gradual for $t=1.01$ and $t=1.07$. At zero or small voltages, $\langle\phi\rangle$ is smaller than the bulk value since the pore is hydrophobic $\left(a^{2} \Delta \gamma=-0.4\right)$. For voltages below $\sim-0.22 \mathrm{~V}$ water becomes the majority component in the pore, $\phi>0.5$ $\left(n_{0}=0.01 \mathrm{M}\right)$. For $n_{0}=0.1 \mathrm{M}$ mixtures this occurs at smaller value of $\left|\psi_{s}\right|$. The bulk composition is $\phi_{0}=0.3$ and $D=5$ nm.

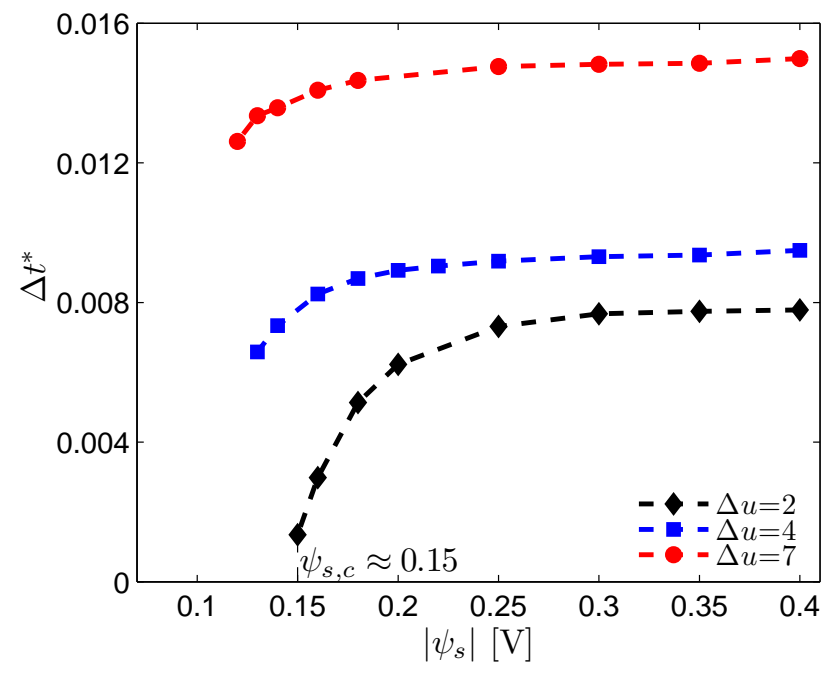

FIG. 4. The temperature shift $\Delta t^{*}$ as a function of the wall potential $\psi_{s}$ for a hydrophobic pore and different values of $\Delta u^{ \pm}$. Here $t=0.96, a^{2} \Delta \gamma=-0.4$ and $D=10 \mathrm{~nm}$. Lines are a guide to the eye. each curve) decreases with increasing $\Delta u$, although not dramatically. Above the critical voltage $\Delta t^{*}$ increases with $\left|\psi_{s}\right|$ until it saturates. This saturation occurs more quickly with large ionic selectivity (large values of $\Delta u$ ).

In summary, we use a simple modified PoissonBoltzmann theory to describe the thermodynamics of porous membranes immersed in aqueous mixtures. We show that pore filling transitions are influenced by the confinement of the pore and occur at temperatures significantly above the so-called precipitation temperatures of mixtures with ions without the pore confinement. The theory can be used to predict whether the pore is filled with the cosolvent-rich or with the water-rich phases.

Fig. 4 and Fig. 3 show abrupt and gradual filling transitions with increasing pore potential, respectively; equivalently this means sensitivity to the pore's surface charge. For membranes with immobile charges the surface heterogeneity leads to variations of the surface charge. In such membranes some pores will be "open" while some will be "closed" and the conductance of liquids or solutes across the membrane will be heterogeneous accordingly.

For composite porous carbonaceous membranes and other types of solid membranes, Fig. 3 and Fig. 4 demonstrate the feasibility of robustly and sensitively filling or emptying the pore with water in an on/off manner by connecting the membrane to an external potential. This filling will lead to reversible blockage or clearance of the membrane to small solutes, depending on their hydrophobicity or hydrophilicity.

The continuous filling of a very hydrophobic pore at temperatures up to $20 \mathrm{~K}$ above $T_{c}$ in Fig. 3 suggests that our predictions could be tested, for example, using water-acetonitrile at room temperature.

S. S. acknowledges funding from the European Unions Horizon 2020 research and innovation programme under the Marie Skłodowska-Curie grant agreement No. 656327. Y. T. acknowledges the support from COST Action MP1106 and Israel Science Foundation Grant No. $56 / 14$.

\section{Supplementary material}

Profiles. In equilibrium the profiles $\phi(z), \psi(z)$ and $n^{ \pm}(z)$ are the extremizers of the grand potential, and thus they obey 


$$
\begin{aligned}
\frac{\delta \Omega}{\delta \phi}= & -C \nabla^{2} \phi+\frac{k_{B} T}{v_{0}}\left[\log \left(\frac{\phi}{1-v_{0} n^{+}-v_{0} n^{-}-\phi}\right)+\chi\left(1-2 \phi-v_{0} n^{+}-v_{0} n^{-}\right)\right] \\
& -\frac{1}{2} \frac{d \varepsilon}{d \phi}(\nabla \psi)^{2}-k_{B} T\left(\Delta u^{+} n^{+}+\Delta u^{-} n^{-}\right)-\mu / v_{0}=0 \\
\frac{\delta \Omega}{\delta \psi}= & \nabla \cdot(\varepsilon(\phi) \nabla \psi)+\left(n^{+}-n^{-}\right) e=0 \\
\frac{\delta \Omega}{\delta n^{ \pm}}= & \pm e \psi+k_{B} T\left[\log \left(\frac{v_{0} n^{ \pm}}{1-v_{0} n^{+}-v_{0} n^{-}-\phi}\right)-\left(\Delta u^{ \pm}+\chi\right) \phi\right]-\lambda^{ \pm}=0 .
\end{aligned}
$$

$n^{ \pm}$in Eq. (S.3) can be isolated to give

$$
n^{ \pm}=\frac{P^{ \pm}(1-\phi)}{v_{0}\left(1+P^{+}+P^{-}\right)},
$$

where $P^{ \pm}$is given by

$$
P^{ \pm}=\exp \left(\frac{\mp e \psi}{k_{B} T}+\left(\Delta u^{ \pm}+\chi\right) \phi+\frac{\lambda^{ \pm}}{k_{B} T}\right) .
$$

One can put Eq. (S.4) in Eqs. (S.1) and (S.2) and solve to obtain the electrostatic potential and composition profiles. From these profiles we determine all other quantities. In the Poisson-Boltzmann limit of point-like ions, $v_{0} n^{+}, v_{0} n^{-} \rightarrow 0$, Eq. (S.1) reduces to

$$
\begin{aligned}
& -C \nabla^{2} \phi+\frac{\partial f_{m}}{\partial \phi}-\frac{1}{2} \frac{\mathrm{d} \varepsilon}{\mathrm{d} \phi}(\nabla \psi)^{2} \\
& -k_{B} T\left[\Delta u^{+} n^{+}+\Delta u^{-} n^{-}\right]-\mu / v_{0}=0,
\end{aligned}
$$

and the ion densities follow the Boltzmann distributions

$$
n^{ \pm}=n_{0} e^{\mp e \psi / k_{B} T+\Delta u^{ \pm}\left(\phi-\phi_{0}\right)} .
$$

Determination of the composition $\phi_{t}$. Consider a small arbitrary volume of the mixture within a homogeneous bulk with a composition $\phi_{0}$ and a dissolved salt of pointlike ions with a concentration $n_{0}$. In the absence of external electric fields $(\psi=0)$ or surface fields $\left(a^{2} \Delta \gamma=0\right)$ the composition and ion densities in this volume are also homogeneous. The total grand density in this case, $\omega_{0}$, is written as

$$
\omega_{0}=f_{0}-\lambda^{+} n^{+}+\lambda^{-} n^{-}-\mu \phi / v_{0} .
$$

where $f_{0}=f_{\mathrm{m}}+f_{\mathrm{i}}$ and $\lambda^{ \pm}$and $\mu$ are the chemical potentials of the ions and water, respectively. $f_{\mathrm{i}}$ defined in Eq. (2) of the main text. In the limit of point-like ions, $f_{\mathrm{m}}$ from equation Eq. (1) of the main text reduces to

$$
f_{\mathrm{m}}=\frac{k_{B} T}{v_{0}}[\phi \log (\phi)+(1-\phi) \log (1-\phi)+\chi \phi(1-\phi)]
$$

For hydrophilic ions, $\Delta u^{ \pm}>0$, and a water-poor reservoir, $\phi_{0}<1 / 2, \omega_{0}$ is modified such that a water-rich phase in the small volume may coexist with the waterpoor phase of the reservoir. The composition and salt concentration of each phase are determined by the equality of chemical potentials in the small volume and reservoir:

$$
\begin{gathered}
\mu\left(\phi_{0}, n_{0}\right)=\mu\left(\phi_{h}, n_{h}\right), \\
\lambda^{ \pm}\left(\phi_{0}, n_{0}\right)=\lambda^{ \pm}\left(\phi_{h}, n_{h}\right),
\end{gathered}
$$

where $\phi_{h}$ and $n_{h}$ are the composition and salt concentration of the water-rich phase, respectively. The chemical potentials are found from:

$$
\begin{aligned}
\mu & =v_{0} \frac{\partial f_{0}}{\partial \phi}=k_{B} T\left[\log \left(\frac{\phi}{1-\phi}\right)+\chi(1-2 \phi)\right. \\
& \left.-v_{0}\left(\Delta u^{+} n^{+}+\Delta u^{-} n^{-}\right)\right] \\
\lambda^{ \pm} & =\frac{\partial f_{0}}{\partial n^{ \pm}}=k_{B} T\left[\log \left(v_{0} n^{ \pm}\right)-\Delta u^{ \pm} \phi\right]
\end{aligned}
$$

Using these relations in Eqs. (S.10) and (S.11) we can numerically determine $\phi_{h}$ and $n_{h}$. The water-rich phase is thermodynamically preferable when $\omega_{0}\left(\phi_{h}, n_{h}\right)<$ $\omega_{0}\left(\phi_{0}, n_{0}\right)$. For a fixed value of $n_{0}$, the locus of reservoir compositions $\phi_{t}\left(T ; n_{0}, \Delta u^{ \pm}\right)$for which $\omega_{0}\left(\phi_{h}, n_{h}\right)=$ $\omega_{0}\left(\phi_{0}, n_{0}\right)$ defines a stability diagram in the $\phi_{0}-T$ plane. $\phi_{t}$ is shown for $n_{0}=0.1 \mathrm{M}, \Delta u^{ \pm}=4$, and point-like ions in Fig. 2 of the main text. At $\phi_{0}=\phi_{t}$, a so-called precipitation transition [12] occurs and the composition jumps to $\phi_{h}$ corresponding to $\phi_{t}$.

A similar result was obtained by Okamoto and Onuki 12] within a canonical ensemble calculation, and our treatment corresponds to the limit of zero volume fraction of the water-rich phase in their work. In our work, $\phi_{t}(T)$ is viewed as the boundary in the $\phi_{0}-T$ plane for which the hydrophilic solute alone can induce a phase change inside the pore. In the main text, however, we study phase changes inside pores in the range $\phi_{0}<\phi_{t}(T)$.

[1] O. Kedem and A. Katchalsky,
Biochimica et biophysica acta 27, 229 (1958). 
[2] M. M. Pendergast and E. M. V. Hoek, Energy \& Environmental Science 4, 1946 (2011).

[3] M. Carta, R. Malpass-Evans, M. Croad, Y. Rogan, J. C. Jansen, P. Bernardo, F. Bazzarelli, and N. B. McKeown, Science 339, 303 (2013)

[4] B. Yang $\begin{array}{llll} & \text { T. } & \text { T. }\end{array}$ Journal of Membrane Science 218, 247 (2003), 86.

[5] B. Yameen, M. Ali, R. Neumann, W. Ensinger, W. Knoll, and O. Azzaroni, Small 5, 1287 (2009), 87.

[6] X. Hou, Y. Hu, A. Grinthal, M. Khan, and J. Aizenberg, Nature 519, 70 (2015), 10.

[7] J. Dzubiella, R. J. Allen, and J. P. Hansen, Journal of Chemical Physics 120, 5001 (2004).

[8] J. Dzubiella and J. Hansen, Journal of Chemical Physics 122 (2005), 10.1063/1.1927514

[9] M. R. Powell, L. Cleary, M. Davenport, K. J. Shea, and Z. S. Siwy, Nature Nanotechnology 6, 798 (2011) 81.

[10] S. N. Smirnov, I. V. Vlassiouk, and N. V. Lavrik, Acs Nano 5, 7453 (2011)

[11] Y. Tsori and L. Leibler, Proc. Nat. Acad. Sci. 104, 7348 (2007)

[12] R. Okamoto and A. Onuki, Phys. Rev. E 82, 051501 (2010)

[13] M. Bier, A. Gambassi, and S. Dietrich, J. Chem. Phys. 137, 034504 (2012)

[14] M. E. Leunissen, A. van Blaaderen, A. D. Hollingsworth, M. T. Sullivan, and P. M. Chaikin,
Proc. Natl. Acad. Sci. U.S.A. 104, 2585 (2007)

[15] U. Nellen, J. Dietrich, L. Helden, S. Chodankar, K. Nygård, J. F. van der Veen, and C. Bechinger, Soft Matter 7, 5360 (2011).

[16] S. Samin, M. Hod, E. Melamed, M. Gottlieb, and Y. Tsori, Physical Review Applied 2 (2014), 10.1103/physrevapplied.2.024008

[17] S. Samin and Y. Tsori, EPL 95, 36002 (2011).

[18] R. Okamoto and A. Onuki, Phys. Rev. E 84, 051401 (2011).

[19] S. Samin and Y. Tsori, J. Chem. Phys. 136, 154908 (2012).

[20] F. Pousaneh and A. Ciach, Soft Matter 10, 8188 (2014)

[21] I. Borukhov, D. Andelman, and H. Orland, Phys. Rev. Lett. 79, 435 (1997).

[22] A. Onuki and H. Kitamura, J. Chem. Phys. 121, 3143 (2004).

[23] C. Kalidas, G. Hefter, and Y. Marcus, Chem. Rev. 100, $819(2000)$ Y. Marcus, Chem. Rev. 107, 3880 (2007)

[24] Y. Tsori, Reviews of Modern Physics 81, 1471 (2009).

[25] D. Ben-Yaakov, D. Andelman, D. Harries, and R. Podgornik, J. Phys. Chem. B 113, 6001 (2009). 